\title{
Charging damage during residual metal overetching
}

\author{
Gyeong S. Hwang and Konstantinos P. Giapis ${ }^{\text {a) }}$ \\ Division of Chemistry and Chemical Engineering, California Institute of Technology, Pasadena, \\ California 91125
}

(Received 20 October 1998; accepted for publication 14 December 1998)

\begin{abstract}
The influence of electron and ion temperatures on charging damage during residual metal (latent antenna) overetching in high-density plasmas is investigated by Monte Carlo simulations. The tunneling current through a thin gate oxide, electrically connected to the antenna, increases significantly with electron temperature, mainly as a result of changes in plasma current and ion energy distribution. However, the current decreases with ion temperature as ion shading: (a) directly decreases the ion flux to the antenna and (b) neutralizes the negative charge at the upper mask sidewalls, thus allowing more electrons to enter the pattern. The role of exposed antenna areas (trench bottoms and perimeter) is examined from the perspective of current imbalance. (C) 1999 American Institute of Physics. [S0003-6951(99)02407-9]
\end{abstract}

Metal etching is an essential processing step in the definition of interconnects for integrated circuits. It is typically performed in high-density plasmas, where control of ion energy permits directional pattern transfer of a photoresist mask into the metal layer with sufficient selectivity to preserve linewidth. As critical dimensions shrink and aspect ratios increase, charging damage to buried thin gate oxides connected to the metal lines has been found to occur more frequently. ${ }^{1}$ The damage arises from two main sources: plasma nonuniformity ${ }^{2}$ and electron shading; ${ }^{1}$ it manifests itself as degradation or breakdown of the gate oxide due to tunneling currents flowing in response to potential differences across the oxide. As plasmas have become more uniform, electron shading has emerged as the major cause of charging damage. ${ }^{3}$ Electron shading describes the imbalance of ion and electron currents to the bottom of narrow trenches due to differences in their angular distributions. ${ }^{4}$ Although the physics of charging damage due to electron shading has been proposed, ${ }^{5,6}$ the influence of critical plasma parameters is not understood.

Charging damage in patterned structures exposed to a uniform plasma may occur by means of (a) tunneling current surges at the onset of overetching, ${ }^{6}$ and (b) steady-state tunneling currents during overetching. ${ }^{5,6}$ Overetching is needed due to reactive ion etching lag (RIE-lag), a phenomenon that describes a decrease in etch rate with aspect ratio of the pattern. ${ }^{7}$ Overetching begins when open areas between patterns clear and ends when the narrow trenches between lines are also thoroughly etched. During the early stages of overetching, the lines are connected through the remaining metal at the trench bottoms forming an antenna, as illustrated in Fig. 1. Large tunneling currents are possible during this "latent antenna overetching" period as the imbalance of ion and electron currents to each trench bottom is amplified by the antenna collection area. When the trench bottoms begin to clear, the antenna effect stops and the tunneling current is considerably reduced. In this letter, we focus on charging damage during latent antenna overetching; a treatment of the

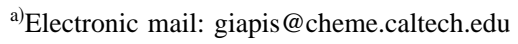

current surges is postponed until they are observed experimentally.

High-density plasmas operate generally at higher electron temperature $\left(T_{e}\right)$ than more traditional low-density glow discharges; their use in polysilicon and metal etching has coincided with frequent observation of charging damage. ${ }^{1}$ The ion temperature $\left(T_{i}\right)$ is also higher in such plasmas, but its role in charging damage has not been reported. In this numerical study, we vary $T_{e}$ between $2-8 \mathrm{~V}$ and $T_{i}$ between $0.1-0.5 \mathrm{~V}$ (typical ranges); since we aim at revealing trends, we shall not worry about what combinations of $T_{e}$ and $T_{i}$ are possible for a particular plasma. We model a fully dissociated, low-pressure ( $<5 \mathrm{mTorr}) \mathrm{Cl}_{2}$ plasma of electron density $1 \times 10^{12} \mathrm{~cm}^{-3}$. The wafer electrode is radio-frequency (rf) biased at $0.4 \mathrm{MHz}$ with a peak-to-peak voltage of $60 \mathrm{~V}$. The pattern consists of five $0.3 \mu \mathrm{m}$ features separated by 0.3 $\mu \mathrm{m}$ spaces. Identical patterns are separated by $4-\mu \mathrm{m}$-wide open areas. At the onset of overetching, each feature consists of a $0.6-\mu \mathrm{m}$-thick photoresist onto a $0.3-\mu \mathrm{m}$-thick metal line, formed on top of a thick $(>100 \mathrm{~nm})$ layer of $\mathrm{SiO}_{2}$. The metal lines are connected by a thin metal layer remaining in the trenches as a result of RIE lag. The center line sits on top of a metallic conduit to a small gate, separated from the grounded substrate by 4 -nm-thick gate oxide (Fig. 1). The antenna ratio, defined here as the area ratio of one trench footprint $\left(A_{t}=\right.$ width $\times$ length $)$ over that of the gate oxide $\left(A_{o}\right)$, is taken to be 1000:1.

The Monte Carlo simulation of microstructure charging is performed as described elsewhere, ${ }^{8}$ explicitly and selfconsistently accounting for charge dissipation along dielectric surfaces ${ }^{9}$ and electron tunneling currents through thin

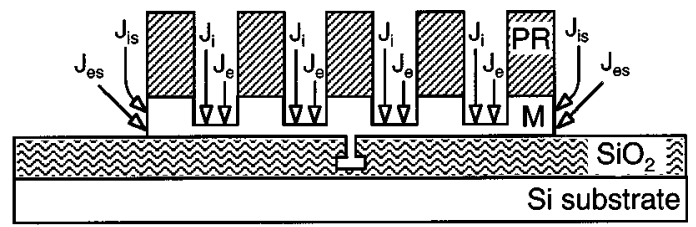

FIG. 1. Schematic of the latent antenna structure at the onset of overetching $(\mathrm{M}=$ metal, $\mathrm{PR}=$ photoresist mask $)$. 


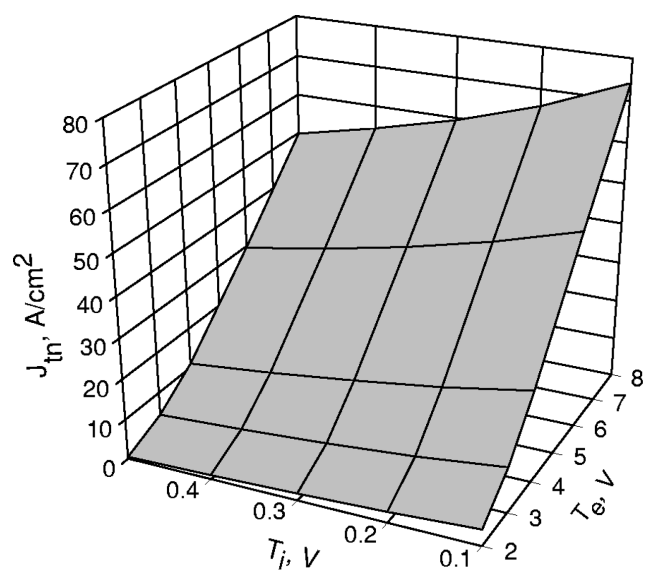

FIG. 2. The steady-state tunneling current through the gate oxide as a function of the electron $\left(T_{e}\right)$ and ion $\left(T_{i}\right)$ temperatures.

gate oxides. ${ }^{4}$ Surface currents are assumed to flow readily when the surface electric field on photoresist or exposed oxide exceeds $1 \mathrm{MV} / \mathrm{cm}^{9}{ }^{9}$ Although the magnitude of the tunneling current is treated as a measure of charging damage, the effects of oxide degradation are not considered.

The dependence of the steady-state tunneling current, $J_{t n}$, through the $4 \mathrm{~nm}$ gate oxide on the electron and ion temperatures is summarized in Fig. 2. Table I also lists representative potential and current values at various locations, as defined in Fig. 1. $J_{t n}$ is the sum of the Fowler-Nordheim and direct tunneling currents, which are calculated from formulas given elsewhere ${ }^{4}$ at steady state, $J_{t n}$ can be also approximated by

$$
J_{t n}=N\left(A_{t} / A_{o}\right)\left(J_{i}-J_{e}\right)+2\left(A_{s} / A_{o}\right)\left(J_{i s}-J_{e s}\right),
$$

where $N$ is the number of trenches in the antenna, $A_{s}$ is the area of the outer antenna sidewall, $J_{i}$ and $J_{e}$ are the ion and electron current densities at a trench bottom, and $J_{i s}$ and $J_{e s}$ are the ion and electron current densities at the outer antenna sidewall.

As expected, $J_{t n}$ depends strongly on $T_{e}$. The current increases by a factor of 18.5 when raising $T_{e}$ from 2 to $8 \mathrm{~V}$ (for $T_{i}=0.1 \mathrm{~V}$ constant). The increase can be even larger, e.g., a factor of 100 for the same rise of $T_{e}$ but at a higher $T_{i}=0.5 \mathrm{~V}$ (see Table I). This dependence confirms again the crucial role of electron shading on charging damage. While small currents at low $T_{e}$ may be tolerated, the exponential increase in current accompanying plasma operation at higher $T_{e}$ is bound to lead to damage. Although not as strong, the dependence of $J_{t n}$ on $T_{i}$ is remarkable. The tunneling current

TABLE I. Calculated ion and electron currents supplied to the residual metal at the bottom of each trench $\left(J_{i}, J_{e}\right)$ and to each outer antenna sidewall $\left(J_{i s}, J_{e s}\right)$ for various combinations of ion $\left(T_{i}\right)$ and electron $\left(T_{e}\right)$ temperatures; the steady-state antenna potential $\left(V_{p}\right)$ and the corresponding tunneling current $\left(J_{t n}\right)$ through a $4 \mathrm{~nm}$ gate oxide (antenna ratio $\left.=1000\right)$ are also listed.

\begin{tabular}{ccrccc}
\hline \hline $\begin{array}{c}T_{e} \\
(\mathrm{~V})\end{array}$ & $\begin{array}{c}T_{i} \\
(\mathrm{~V})\end{array}$ & $\begin{array}{c}J_{i} / J_{e} \\
\left(\mathrm{~mA} / \mathrm{cm}^{2}\right)\end{array}$ & $\begin{array}{c}J_{i s} / J_{e s} \\
\left(\mathrm{~mA} / \mathrm{cm}^{2}\right)\end{array}$ & $\begin{array}{c}V_{p} \\
(\mathrm{~V})\end{array}$ & $\begin{array}{c}J_{t n} \\
\left(\mathrm{~A} / \mathrm{cm}^{2}\right)\end{array}$ \\
\hline 2 & 0.1 & $3.96 / 1.39$ & $0.17 / 3.12$ & 5.85 & 3.99 \\
8 & 0.1 & $22.1 / 3.42$ & $4.89 / 4.85$ & 6.87 & 74.24 \\
2 & 0.5 & $3.31 / 1.82$ & $4.27 / 3.08$ & 5.29 & 0.51 \\
8 & 0.5 & $18.16 / 4.85$ & $4.23 / 5.18$ & 6.72 & 49.81 \\
\hline \hline
\end{tabular}
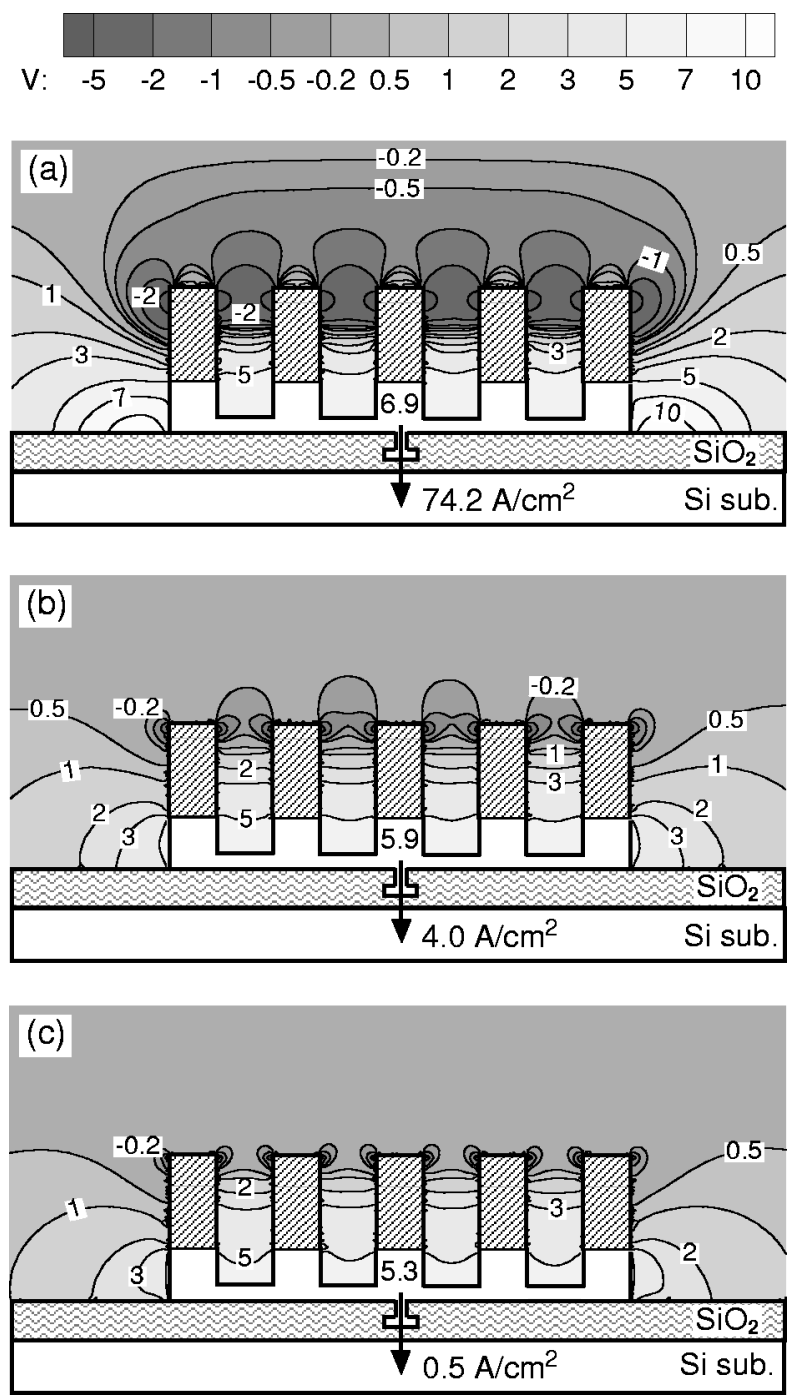

FIG. 3. Charging maps for various combinations of electron and ion temperatures: (a) $T_{e}=8 \mathrm{~V}, T_{i}=0.1 \mathrm{~V}$, (b) $T_{e}=2 \mathrm{~V}, T_{i}=0.1 \mathrm{~V}$, and (c) $T_{e}$ $=2 \mathrm{~V}, T_{i}=0.5 \mathrm{~V}$.

actually decreases roughly by $30 \%-90 \%$ ( $T_{e}$ dependent) upon increasing $T_{i}$ from 0.1 to $0.5 \mathrm{~V}$. This beneficial effect of ion temperature on charging damage has not been discussed before.

To better understand the influence of $T_{e}$ and $T_{i}$ on tunneling current, it is instructive to plot the potential contour maps for various parameter combinations. In Figs. 3(a) and 3(b), we compare a case of "severe" electron shading ( $T_{e}$ $=8 \mathrm{~V})$ to one of "mild" electron shading $\left(T_{e}=2 \mathrm{~V}\right)$, both at a low $T_{i}=0.1 \mathrm{~V}$. As a consequence of the more energetic electrons at higher $T_{e}$, significant negative charging appears at the upper photoresist sidewalls of the former case. One might expect a decrease in the electron current to the trench bottom as a result of the more negative entrance potential. ${ }^{10}$ However, Table I indicates the opposite: $J_{e}$ increases from 1.39 to $3.42 \mathrm{~mA} / \mathrm{cm}^{2}$ upon raising $T_{e}$ from 2 to $8 \mathrm{~V}$, respectively. The proportionality of the plasma conduction current to the Bohm velocity $\left(\propto \sqrt{T_{e}}\right)$ could account for at most a factor of 2 increase if charging of the sidewalls were absent. The factor of 2.5 increase observed is attributed to the larger population of higher energy electrons at $T_{e}=8 \mathrm{~V}$, which can penetrate the more negative entrance potential. Once in the Downloaded 30 Apr 2006 to 131.215.240.9. Redistribution subject to AlP license or copyright, see http://apl.aip.org/apl/copyright.jsp 


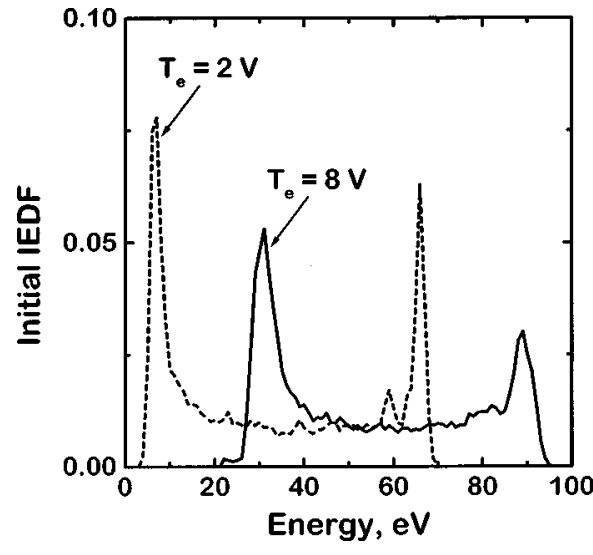

FIG. 4. Initial ion energy distribution functions (IEDF) calculated for the indicated values of electron temperature $\left(T_{e}\right)$. Other plasma parameters were ion temperature $=0.1 \mathrm{~V}$, plasma density $=1 \times 10^{12} \mathrm{~cm}^{-3}$, rf bias $=60 \mathrm{~V}$ (peak-to-peak) applied at $0.4 \mathrm{MHz}$.

trench, such electrons are more readily directed to the bottom by the larger electric field between trench entrance and conductive bottom. Remarkably, the ion current to the trench bottom increases much more: from 3.96 to $22.1 \mathrm{~mA} / \mathrm{cm}^{2}$ (a factor of 5.6!) for the same rise in $T_{e}$. The magnitude of the increase cannot be explained by the change in plasma conduction current. What is missing?

Raising the electron temperature increases the minimum sheath potential, $V_{\mathrm{dc}}$ from 4 to $21.5 \mathrm{~V}$ which, in turn, shifts both the low and high energy peaks of the bimodal ion energy distribution to higher energies, as predicted from a selfconsistent treatment of the sheath ${ }^{11}$ and illustrated in Fig. 4. Since $T_{i}$ remains constant, more energetic ions cause a decrease in ion shading; as a result, fewer ions are lost to the sidewalls by direct irradiation. Since the antenna potential increases by a mere $1 \mathrm{~V}$ (see Table I), fewer ions are deflected away from the bottom. For these two reasons, many more ions make it to the trench bottom at the higher $T_{e}$, thereby contributing to the net tunneling current through the buried gate oxide. It is interesting to note that the worsening in differential charging at higher $T_{e}$ does not really play much of a role: there are just more directional ions arriving at the patterned surface at higher energy. The changes in $J_{i}$ and $J_{e}$, when amplified by the antenna collection area $\left[N\left(A_{t} / A_{o}\right)\right]$, account for a larger increase in $J_{t n}$ than listed in Table I; here is where the antenna perimeter plays a role.

The imbalance of ion and electron currents to the outer conductive sidewalls can be positive or negative, depending on the values of $T_{e}$ and $T_{i}$. As inferred from Table I, a net negative current enters the antenna through the outer sidewalls at $T_{e}=2 \mathrm{~V}$; the net current becomes slightly positive at $T_{e}=8 \mathrm{~V}\left(T_{i}=0.1 \mathrm{~V}\right.$ constant $)$ as a result of the large increase in $J_{i s}$ which far exceeds the increase in $J_{e s}$ due to the dependence of the plasma conduction current on $T_{e}$. This result is surprising considering that the ions become more directional at $T_{e}=8 \mathrm{~V}$. Its origin lies in the significant negative charging at the upper mask sidewall facing the open area [Fig. 3(a)].
The negative potential is large enough to deflect slower ions that travel in the vicinity of the mask toward the antenna.

The ion temperature effect can be explained by the broadening of the ion angular distribution which (a) reduces the ion flux to the trench bottom (ion shading), and (b) increases ion bombardment of the upper mask sidewalls, thus reducing the negative potential at the trench entrance [Fig. $3(\mathrm{c})$ ]. Indeed, this is most easily observed at the outer antenna sidewalls where the ion flux increases from 0.17 to $4.27 \mathrm{~mA} / \mathrm{cm}^{2}$ when $T_{i}$ is changed from 0.1 to $0.5 \mathrm{~V}$, respectively ( $T_{e}=2 \mathrm{~V}$ constant), although the negative potential at the upper mask sidewalls actually decreases. Furthermore, $J_{i}$ decreases from 3.96 to $3.31 \mathrm{~mA} / \mathrm{cm}^{2}$ for the same change. Remarkably, $J_{e}$ increases slightly from 1.39 to $1.82 \mathrm{~mA} / \mathrm{cm}^{2}$, indicating that more electrons penetrate the reduced entrance potential. The net result of the $T_{i}$ increase is a dramatic decrease in $J_{t n}$ from 4.0 to $0.5 \mathrm{~A} / \mathrm{cm}^{2}$. The trend also holds for larger values of $T_{e}$ (Fig. 2).

In conclusion, self-consistent charging simulations provide insight into the current balances that influence charging damage during residual metal overetching. The steady-state tunneling current to a buried gate oxide was found to depend strongly on electron temperature, not because of more severe differential charging of the pattern but rather as a result of the influence of the electron temperature on the ion energy distribution function and on the plasma conduction current. Increasing ion temperature decreased the tunneling current in all cases suggesting a reduction in charging damage. The net current collected by the antenna under all conditions was proportional to the total area between the antenna fingers. The antenna perimeter was found to play a more complex role whose significance depended on both electron and ion temperatures. The simulation results suggest that operation at low electron and high ion temperatures, e.g., by running the plasma at higher pressure, should be preferred for a reduction in charging damage during overetching.

This material was based on work partially supported by NSF (ECS-9729968) and a Camille Dreyfus Teacher-Scholar Award to K.P.G. An Applied Materials Scholarship to G.S.H. is gratefully acknowledged.

\footnotetext{
${ }^{1}$ K. Hashimoto, Jpn. J. Appl. Phys., Part 1 32, 6109 (1993).

${ }^{2}$ C. T. Gabriel and J. P. McVittie, Semicond. Sci. Technol., 81 (1992).

${ }^{3} \mathrm{~V}$. Vahedi, N. Benjamin, and A. Perry, Proceedings of the 2nd International Symposium on Plasma Process-Induced Damage (NCCAVS, Sunnyvale, CA, 1997), p. 41.

${ }^{4}$ K. P. Giapis and G. S. Hwang, Jpn. J. Appl. Phys., Part 1 37, 2281 (1998).

${ }^{5}$ T. Kinoshita, S. Krishnan, W. Dostalik, and J. McVittie, Proceedings of the 2nd International Symposium on Plasma Process-Induced Damage (NCCAVS, Sunnyvale, CA, 1997), p. 45.

${ }^{6}$ G. S. Hwang and K. P. Giapis, J. Electrochem. Soc. 144, L285 (1997).

${ }^{7}$ R. A. Gottscho, C. W. Jurgensen, and D. J. Vitkavage, J. Vac. Sci. Technol. B 10, 2133 (1992).

${ }^{8}$ G. S. Hwang and K. P. Giapis, J. Vac. Sci. Technol. B 15, 70 (1997).

${ }^{9}$ G. S. Hwang and K. P. Giapis, J. Appl. Phys. 84, 683 (1998).

${ }^{10}$ T. Kinoshita and J. McVittie, Proceedings of the 3nd International Symposium on Plasma Process-Induced Damage (NCCAVS, Sunnyvale, CA, 1998), p. 172.

${ }^{11}$ M. A. Lieberman, IEEE Trans. Plasma Sci. 16, 638 (1988).
} 